\title{
Global exponential regulation of discrete time linear systems with unknown neutrally stable exosystems
}

\author{
Riccardo Marino, Giovanni L. Santosuosso* \\ Dipartimento di Ingegneria Elettronica, Università di Roma “Tor Vergata”, Via del Politecnico 1, 00133 Roma, Italy
}

\section{A R T I C L E I N F O}

\section{Article history:}

Received 5 February 2010

Received in revised form 19 April 2011

Accepted 21 April 2011

Available online 24 May 2011

\section{Keywords:}

Adaptive control

Disturbance rejection

Regulation

\begin{abstract}
A B S T R A C T
Discrete time, linear, stabilizable and detectable systems with known parameters are considered: the regulation problem is addressed when the reference output and/or the disturbances contain sinusoidal terms generated by a linear exosystem with unknown parameters. Only an upper bound on the number of unknown sinusoids is supposed to be known. A constructive algorithm is proposed to drive the regulation error exponentially to zero on the basis of its measurement only, under the same necessary and sufficient conditions which are required when the exosystem is known. The control strategy includes an online detector for the number of excited frequencies and exponentially converging global estimates of the exosystem unknown parameters. An illustrative example containing a variable number of frequencies is worked out and simulated.
\end{abstract}

(C) 2011 Elsevier B.V. All rights reserved.

\section{Introduction}

The output regulation problem for linear systems in the presence of unknown harmonic disturbances and/or references has attracted a considerable interest in the control community. In the framework of the regulation theory introduced in [1], the control goal is to regulate to zero the output tracking error of a given system which is perturbed by nonvanishing unknown modes generated by a neutrally stable linear exosystem. This research problem is motivated by many applications such as active suspension design [2], disk drive speed regulation [3], eccentricity compensation [4], active noise control [5], harmonic elimination in power electronics [6], feedback control of vibrations in helicopters $[7,8]$.

In the discrete time setting, under the minimum phase assumption (MP) on the perturbed system, it is shown in [9] that unknown deterministic disturbances generated by exosystems with eigenvalues on the unit circle can be compensated via the model reference adaptive control techniques described in [10], yielding asymptotic convergence to zero of the output regulation error. This task is accomplished via the controller adaptation with respect to both the system and the exosystem unknown parameters. A different approach is followed [11-13] for systems which are allowed to be non-minimum phase (NMP): it focuses on the construction of regulation algorithms that are adaptive with respect to the unknown exosystem parameters only, under the assumption that the system parameters are known. The Youla

\footnotetext{
* Corresponding author. Tel.: +39 0672597414.

E-mail addresses: marino@ing.uniroma2.it (R. Marino), santosuosso@ing.uniroma2.it (G.L. Santosuosso).
}

parametrization of stabilizing controllers is used to construct a regulator by introducing the additional hypothesis that a solution to the regulation problem exists when the controller stable transfer function is restricted to have all poles in zero. In this special case, the regulation is obtained by tuning the Youla transfer function numerator coefficients. In [14] a different approach is followed, presenting a discrete time adaptive algorithm to reject a known number of sinusoidal disturbances with unknown frequencies; the strategy in [14] is tested on an active noise control testbed, showing its practical implementability when the system model is partially known; the regulation error is shown to convergence to zero locally and exponentially via averaging theory.

Currently in the discrete time setting, the exponential regulation problem of a linear system allowed to be NMP perturbed by an unknown neutrally stable exosystem is still unsolved, without requiring additional assumptions besides the necessary hypotheses for a solution to exist when the exosystem's parameters are known. In this paper we solve this problem, designing an algorithm that improves previous results. The necessary hypotheses for a solution to exist are shown to be also sufficient for the controller construction achieving exponential regulation even when the exosystem is unknown. In fact, only an upper bound of the number of the unknown harmonics generated by the exosystem is required to obtain exponential regulation. This is achieved via an online detection algorithm for the number of unknown excited frequencies, which allows for the parameters of the minimal exosystem to be exponentially estimated. The harmonic compensation algorithm can be coupled with any robust stabilizing controller, thus improving its performance in the presence of disturbances that contain unknown harmonic signals. 
An additional motivation arises also from the regulation of continuous time linear systems. In fact, in [15], following previous results in [16], the continuous time counterpart of the problem addressed here is solved (see also related work for continuous time linear systems in [17-19] and continuous time nonlinear systems in [20-23]) by a nonlinear control algorithm. Its implementation via fast digital devices generates approximation errors and consequently the regulation error does not converge to zero; the resulting error is also hard to quantify. On the other hand, any continuous time linear system perturbed by a neutrally stable exosystem admits an exact discrete time model if properly coupled with $\mathrm{A} / \mathrm{D}$ and $\mathrm{D} / \mathrm{A}$ devices. Hence, a discrete time algorithm driving to zero the output of the discretized model, drives to zero the original continuous time output at sampling times.

Motivated by the above arguments, in this note we describe a discrete time algorithm which differs from the continuous time one [15] on important aspects. Its estimation strategy is based on the interpretation of the unknown exosystem parameters as additional system states. Since they are multiplied by the entries of the exosystem state vector, the exosystem itself is regarded as a nonlinear system for which we construct a nonlinear observer. A discrete time adaptive observer is designed on the basis of a discrete time 'canonical' class which has a different structure with respect to the one associated with the continuous time problem in [15], yielding an entirely different disturbance estimation algorithm. Thus, we depart from the filters structure in [15] to demonstrate, for the different discrete time filters, the persistency of excitation condition implying exponential regulation.

The paper is organized as follows: in Section 2 the addressed problem is precisely stated. In Section 3 the regulation strategy is outlined. In Section 4 the design of an adaptive harmonic compensation algorithm is described, which solves the problem addressed in this paper. The algorithm is tested and illustrated on a numerical example in Section 5. The conclusions are drawn in Section 6 and in the Appendix the proofs of intermediate results are reported.

\section{Problem statement}

The class of linear systems

$\left\{\begin{array}{l}x(k+1)=A x(k)+b u(k)+P w(k) ; \quad x(0)=x_{0} \\ w(k+1)=R w(k) ; \quad w(0)=w_{0} \\ e(k)=c x(k)+q w(k) ;\end{array}\right.$

is considered, where $(A, b, c)$ is the triple associated to the system to be regulated, with state $x \in \Re^{n}$ and initial condition $x(0)=$ $x_{0}$. The vector $w \in \mathfrak{R}^{r}$ is the exosystem state that generates both the disturbances $P w$ to be rejected and the signal $-q w$ to be tracked by the scalar output $c x$; we assume that $R$ is neutrally stable, with unknown simple eigenvalues on the unit circle, i.e. all the eigenvalues have multiplicity of one. In this paper we are concerned with the design of a feedback control $u$ that drives exponentially to zero the output $e \in \mathfrak{R}$ on the basis of its measurement only. If both the triple $(A, b, c)$ and the triple $(P, R, q)$ are known, following [1] the necessary and sufficient conditions for the solution of the regulator problem are $\left(I_{j} \in \Re^{j} \times \Re^{j}\right.$ denotes the identity matrix of order $j$ )

(H1) the pair $(A, b)$ is stabilizable (i.e. $\operatorname{rank}\left(A-\lambda I_{n}, b\right)<n$ implies $|\lambda|<1)$;

(H2) the pair $(A, c)$ is detectable, (i.e. $\operatorname{rank}\left(A^{T}-\lambda I_{n}, c^{T}\right)<n$ implies $|\lambda|<1)$;

(H3) $\operatorname{rank}\left(\begin{array}{ll}A-\lambda I_{n} & b \\ c & 0\end{array}\right)=n+1$ for any eigenvalue $\lambda$ of the neutrally stable matrix $R$.
Notice that $(\mathrm{H} 1)$ and $(\mathrm{H} 2)$ are the necessary hypotheses for the existence of an output feedback stabilizing controller for the triple $(A, b, c)$. The assumption ( $\mathrm{H} 3)$ requires that no eigenvalue of the matrix $R$ is a zero of the transfer function $c(z I-A)^{-1} b$. By virtue of (H1)-(H3) (see [1]) there exists a unique matrix $\Gamma \in$ $\mathfrak{R}^{n} \times \mathfrak{R}^{r}$ and a unique vector $\gamma \in \mathfrak{R}^{r}$ which solve the regulator equations

$\Gamma R=A \Gamma+b \gamma+P ; \quad c \Gamma+q=0$.

The pair $(\Gamma, \gamma)$ generates the signals $x_{r}=\Gamma w$ and $u_{r}=\gamma w$ which are the references for $x$ and $u$ respectively, since by virtue of (1), (2) $x_{r}(k+1)=A x_{r}(k)+b u_{r}(k)+P w(k)$ and $c x_{r}(k)+q w(k)=$ 0 . The coordinate transformation $\tilde{x}=x-\Gamma w$ yields an error system

$\tilde{x}(k+1)=A \tilde{x}(k)+b\left(u(k)-u_{r}(k)\right)$

$e(k)=c \tilde{x}(k)$,

in which the reference input $u_{r}=\gamma w$ satisfies the "matching condition" and is generated by the exosystem $w(k+1)=R w(k)$. Thus by (H1)-(H3) the regulation of system (1) is achieved by a control law which simultaneously stabilizes the error system (3) and reproduces the reference input $u_{r}(k)$, thus leading to the following regulation problem:

Problem 2.1. Consider the system (1); assume that the triple $(A, b, c)$ is known and the triple $(P, R, q)$ is unknown, with $R$ having known dimensions and simple eigenvalues on the unit circle. If (H1)-(H3) hold, design a dynamic feedback controller such that the closed loop system output $e(k)$ is driven exponentially to zero for any initial condition $x(0) \in \mathfrak{R}^{n}, w(0) \in \mathfrak{R}^{r}$ of system (1).

Remark 2.1. Notice that in the problem statement above it is not specified how many modes are excited by the exosystem actual initial condition $w(0) \in \mathfrak{R}^{r}$. Thus the only information available on $u_{r}(k)$ is simply an upper bound on the number of its harmonics.

\section{Stabilizing regulator design}

Since by $(\mathrm{H} 2)$ the pair $(A, c)$ is detectable, operating a Kalman decomposition with respect to the observable part, whose dimension is $n_{0} \leq n,(3)$ is transformed into

$$
\begin{aligned}
& \left\{x_{u}(k+1)=A_{u} x_{u}(k)+A_{u o} x_{o}(k)+b_{u}\left(u(k)-u_{r}(k)\right)\right. \\
& \left\{\begin{array}{l}
x_{o}(k+1)=A_{o} x_{o}(k)+b_{o}\left(u(k)-u_{r}(k)\right) \\
e(k)=c_{0} x_{o}(k)
\end{array}\right.
\end{aligned}
$$

by a linear change of coordinates $\left[x_{u}^{T}, x_{0}^{T}\right]^{T}=T \tilde{x}$, where $x_{u} \in \mathfrak{R}^{n-n_{o}}$ and $x_{0} \in \Re^{n_{0}}$ are associated to the unobservable and observable parts respectively; $A_{u}, A_{u o}, A_{o}, b_{u}, b_{0}, c_{o}$ are known matrices and vectors. By construction the eigenvalues of matrix $A_{u}$ lie inside the unit circle, the triple $\left(A_{0}, b_{0}, c_{0}\right)$ is observable and stabilizable, with transfer function

$$
W_{o}(z)=p_{b}(z) / p_{o}(z)=c_{o}\left(z I_{n_{o}}-A_{o}\right)^{-1} b_{o} .
$$

The reference input $u_{r}(k)$ may contain two kind of modes: those associated with $r_{1}<r$ eigenvalues of the matrix $R$, located in known positions, such as \pm 1 and those expressed as a sum of unbiased sinusoidal signals with unknown frequencies. Operating a decomposition with respect to these two components, by using the notation

$$
\begin{aligned}
\mathcal{A}_{j} & =\left[\begin{array}{cc}
0 & I_{j-1} \\
0 & 0
\end{array}\right]_{j \times j}, \quad \mathcal{C}_{j}=\left[\begin{array}{llll}
1 & 0 & \cdots & 0
\end{array}\right]_{1 \times j}, \\
\mathscr{B}_{j} & =\left[\begin{array}{llll}
0 & \cdots & 0 & 1
\end{array}\right]_{1 \times j}^{T},
\end{aligned}
$$


with $j$ being a positive integer, the reference input $u_{r}(k)$ can be expressed, without loss of generality, as the output of the exosystem with states $w_{1} \in \mathfrak{R}^{r_{1}}, w_{2} \in \mathfrak{R}^{r_{2}}$,

$w_{1}(k+1)=\left[\mathcal{A}_{r_{1}}-\beta \mathcal{C}_{r_{1}}\right] w_{1}(k), \quad w_{1}(0) \in \mathfrak{R}^{r_{1}}$

$w_{2}(k+1)=R_{2} w_{2}(k), \quad w_{2}(0) \in \Re^{r_{2}}$

$u_{r}(k)=\mathcal{C}_{r_{1}} w_{1}(k)+\mathcal{C}_{r_{2}} w_{2}(k)$

where $\mathcal{C}_{r_{1}} w_{1}(k)$ contains the modes associated with the $r_{1}$ known eigenvalues that are the roots of the polynomial $p_{\beta}(z)=z^{r_{1}}+$ $\left[z^{r_{1}-1}, \ldots, z, 1\right] \beta$, with $\beta \in \Re^{r_{1}}$ and $\mathcal{C}_{r_{2}} w_{2}(k)$ is generated by the modes of $R_{2}$, having unknown eigenvalues with nonzero imaginary part.

The first step in the controller design is to introduce the known mode compensator driven by the new control input $v(k)$

$$
\left\{\begin{array}{l}
s(k+1)=\left[\mathcal{A}_{r_{1}}-\beta \mathcal{C}_{r_{1}}\right] s(k)+\mathscr{B}_{r_{1}} v(k) \\
u(k)=\mathcal{C}_{r_{1}} s(k)
\end{array}\right.
$$

with state $s(k) \in \mathfrak{R}^{r_{1}}$ and arbitrary initial condition $s(0) \in \mathfrak{R}^{r_{1}}$. Notice that

$\operatorname{rank}\left(\begin{array}{ll}{\left[\mathcal{A}_{r_{1}}-\beta \mathcal{C}_{r_{1}}\right]-\lambda I_{r_{1}}} & \mathcal{B}_{r_{1}} \\ \mathcal{C}_{r_{1}} & 0\end{array}\right)=r_{1}+1$

for any eigenvalue $\lambda$ of the matrix $R_{2}$ by construction and that system (9) is reachable and observable with transfer function equal to $1 / p_{\beta}(z)$. Hence, there exists a unique matrix $\Gamma_{2} \in \mathfrak{R}^{r_{1}} \times \mathfrak{R}^{r_{2}}$ and a unique vector $\gamma_{2} \in \Re^{r_{2}}$ which solve the regulator equations

$$
\Gamma_{2} R_{2}=\left[\mathcal{A}_{r_{1}}-\beta \mathcal{C}_{r_{1}}\right] \Gamma_{2}+\mathscr{B}_{r_{1}} \gamma_{2} ; \quad \mathcal{C}_{r_{1}} \Gamma_{2}=\mathcal{C}_{r_{2}} \text {. }
$$

By defining the harmonic signal $\delta=\gamma_{2} w_{2}$ and the vector $x_{e} \in \Re^{N}$, with $N=n_{o}+r_{1}$, as $x_{e}=\left[x_{o}^{T},\left(s-w_{1}-\Gamma_{2} w_{2}\right)^{T}\right]^{T}$, recalling (10), the extended system (5), (8), (9) is

$$
\left\{\begin{array}{l}
x_{e}(k+1)=A_{e} x_{e}(k)+b_{e}(v(k)-\delta(k)) \\
e(k)=c_{e} x_{e}(k)
\end{array}\right.
$$

where

$$
\begin{aligned}
& A_{e}=\left[\begin{array}{ll}
A_{o} & b_{o} \mathcal{C}_{r_{1}} \\
0 & {\left[\mathcal{A}_{r_{1}}-\beta \mathcal{C}_{r_{1}}\right]}
\end{array}\right] ; \quad b_{e}=\left[\begin{array}{l}
0 \\
\mathcal{B}_{r_{1}}
\end{array}\right] ; \\
& c_{e}=\left[\begin{array}{ll}
c_{o} & 0
\end{array}\right] \text {. }
\end{aligned}
$$

We focus now on the stabilizing part of the controller action. By virtue of (H1)-(H2) the undisturbed system, which is obtained by setting in system $(11) \delta(k)=0$, is globally exponentially stabilizable; i.e. there exists an output feedback controller

$$
\left\{\begin{array}{l}
X(k+1)=L_{1} X(k)+L_{2} e(k) \\
v_{S}(k)=H_{1} X(k)+H_{2} e(k)
\end{array}\right.
$$

with state $X \in \Re^{n_{1}}$, such that the origin $x_{e}=0, X=0$ of the closed loop autonomous system obtained from (11), (13) by setting $\delta(k)=0$, and $v=v_{S}$ is globally exponentially stable. Thus, the key part of the regulator construction is the synthesis of an harmonic compensator that generates an estimate $v_{\delta}(k)$ of the unknown harmonic unbiased signal $\delta(k)$. The overall compensating control generates the signal

$v(k)=v_{S}(k)+v_{\delta}(k)$.

Remark 3.1. The regulation algorithm separates the task of stabilization from the one of exact disturbance estimation, in order to preserve the internal stability of the regulated system, which is allowed to be non-minimum phase. To achieve the exact disturbance estimation, the exact knowledge of the regulated system parameters is crucial. However, the harmonic compensation algorithm can be coupled with any 'robust' stabilizing controller in the form (13), in order to improve its performance when the disturbances acting on system (11) contain predominantly harmonic components.

\section{Harmonic compensator design}

The harmonic signal $\delta(k)=\gamma_{2} w_{2}(k)$ is the output of the exosystem $w_{2}(k+1)=R_{2} w_{2}(k)$ in (8) for a suitable initial condition $w_{2}(0) \in \Re^{r_{2}}$ exciting a subset of its modes. Thus $\delta(k)$ is a sum $m$ of sinusoids, with $0 \leq m \leq M$, where $M \leq\left\lfloor r_{2} / 2\right\rfloor \leq\lfloor r / 2\rfloor$ is an upper bound related to the dimension $r$ of the exosystem in (1). We describe the main steps to construct an adaptive observer of the system composed by (11) and a minimal exosystem model yielding $\delta(k)$, assuming that the integer $m$ is known and different from zero. The resulting algorithm will be generalized later by replacing $m$ by a suitable estimate.

\subsection{An adaptive observer with known number of harmonics}

We introduce a linear filter that provides exponential estimates of the state $x_{e}(k)$ in (11) when the sinusoidal disturbance $\delta(k)$ is identically zero. To this purpose, let $a_{i} \in \mathfrak{R}^{+}, 1 \leq i \leq N$, be design parameters such that the polynomial

$p_{a}(z)=z^{N}+a_{1} z^{N-1}+\cdots+a_{N-1} z+a_{N}$

has all its roots inside the unit circle. Let $g_{s} \in \Re^{n_{0}}$ be such that $\operatorname{det}\left[z I_{N}-\left(A_{e}+g_{s} c_{e}\right)\right]=z^{N}+a_{1} z^{N-1}+\cdots+a_{N-1} z+a_{N}$. Consider the linear filter with state $\widehat{x}_{e}(k) \in \mathfrak{R}^{N}$

$\left\{\begin{array}{l}\widehat{x}_{e}(k+1)=\left[A_{e}+g_{s} c_{e}\right] \widehat{x}_{e}(k)-g_{s} e(k)+b_{e} v, \\ y(k)=e(k)-c_{e} \widehat{x}_{e}(k)\end{array}\right.$

and arbitrary initial condition $\widehat{x}_{e}(0) \in \mathfrak{R}^{N}$. By setting $\bar{x}_{e}=x_{e}-\widehat{x}_{e}$, from (5), (16), we obtain

$\left\{\begin{array}{l}\bar{x}_{e}(k+1)=\left[A_{e}+g_{s} c_{e}\right] \bar{x}_{e}(k)-b_{e} \delta(k), \quad \bar{x}_{e}(0) \in \mathfrak{R}^{N} \\ y(k)=c_{e} \bar{x}_{e}(k) .\end{array}\right.$

The error dynamics state $\bar{x}_{e}(k) \in \Re^{N}$ and the output $y(k)$ of system (17) are bounded because (17) is a stable filter driven by the harmonic input $\delta(k)$. The transfer function $W_{e}(z)$ of system (17) from $\delta(k)$ to $y(k)$ is

$W_{e}(z)=-p_{b}(z) / p_{a}(z)$

where $p_{a}(z)$ is the polynomial defined in (15) chosen by the designer and the polynomial $p_{b}(z)$ is the numerator of the transfer function $W_{o}(z)$ in $(6)$.

If $m \neq 0$, then $\delta(k)=\sum_{h=1}^{m} \bar{\sigma}_{h} \sin \left(\omega_{h} k+\bar{\varphi}_{k}\right)$, where $0<$ $\omega_{h}<2 \pi$ are unknown positive real numbers such that $\exp \left( \pm i \omega_{h}\right)$ are among the eigenvalues of the exosystem matrix $R$, while $\bar{\sigma}_{h}$ and $\bar{\varphi}_{k}$ are suitable amplitudes and phases, respectively. The class of linear exosystems that generate the disturbance $\delta(k)$ are associated to matrices whose characteristic polynomial is $p_{\delta}(z)=$ $\prod_{i=1}^{m}\left[z^{2}-2\left(\cos \omega_{i}\right) z+1\right]$. By expanding the terms in $p_{\delta}(z)$ we obtain

$$
\begin{aligned}
p_{\delta}(z)= & \prod_{i=1}^{m}\left[z^{2}-2\left(\cos \omega_{i}\right) z+1\right] \\
= & z^{2 m}+\theta_{1} z^{2 m-1}+\cdots+\theta_{m-1} z^{m+1}+\theta_{m} z^{m} \\
& +\theta_{m-1} z^{m-1}+\cdots+\theta_{2} z^{2 m-2}+\theta_{1} z+1
\end{aligned}
$$

which depends on the $m$ entries of the vector $\theta=\left[\theta_{1}, \theta_{2}, \ldots, \theta_{m}\right]^{T}$, that in turn is a function of the $m$ constants $\cos \omega_{1}, \cos \omega_{2}, \ldots$, $\cos \omega_{m}$. By setting

$S(\theta)=\mathcal{A}_{2 m}-\left[\theta_{1}, \theta_{2}, \ldots, \theta_{m-1}, \theta_{m}, \theta_{m-1}, \ldots, \theta_{2}, \theta_{1}\right]_{2 m}^{T} \mathcal{C}_{2 m}$

the disturbance $\delta(k)$ can be modelled as the output of the following (2m)-order linear excited harmonics minimal exosystem

$$
\left\{\begin{array}{l}
\bar{w}(k+1)=S(\theta) \bar{w}(k), \quad \bar{w}(0) \in \mathfrak{R}^{2 m} \\
\delta(k)=\mathcal{C}_{2 m} \bar{w}(k),
\end{array}\right.
$$


with state $\bar{w}(k) \in \Re^{2 m}$, suitable initial condition $\bar{w}(0) \in \Re^{2 m}$ that excites all the modes of the matrix $S(\theta)$, parametrized by the vector $\theta \in \mathfrak{R}^{m}$. The eigenvalues of the extended system (17), (20) are the roots of the polynomial $p_{a}(z) \cdot p_{\delta}(z)$, where $p_{a}(z)$ and $p_{\delta}(z)$ are the polynomials defined in (15) and (19), respectively. Define $V_{[m]}(\theta) \in \mathfrak{R}^{N+2 m}$ such that

$p_{a}(z) \cdot p_{\delta}(z)=z^{N+2 m}+\left[z^{N+2 m-1}, z^{N+2 m-2}, \ldots, z, 1\right] V_{[m]}(\theta)$.

The vector $V_{[m]}(\theta)$ is a linear combination of the entries of $\theta$. In order to describe a formula expressing in a compact notation the vector $V_{[m]}(\theta)$, consider the vector $\alpha_{[m]} \in \mathfrak{R}^{N+2 m}$

$\alpha_{[m]}=\left[0, \ldots, 0,1, a_{1}, \ldots, a_{N}\right]^{T}$

and define the $m+1$ vectors $\widehat{\alpha}_{[m]}\{j\} \in \Re^{N+2 m}, j=0,1, \ldots, m$, according to the formulae

$\left\{\begin{array}{l}\widehat{\alpha}_{[m]}\{j\}=\left[\left(\mathcal{A}_{N+2 m}\right)^{j}+\left(\mathcal{A}_{N+2 m}\right)^{2 m-j}\right] \alpha_{[m]}, \\ \quad \text { for all } 0 \leq j \leq m \\ \widehat{\alpha}_{[m]}\{m\}=\left(\mathcal{A}_{N+2 m}\right)^{m} \alpha_{[m]},\end{array}\right.$

then $V_{[m]}(\theta)=\widehat{\alpha}_{[m]}\{0\}+\widehat{\alpha}_{[m]} \theta$ where $\widehat{\alpha}_{[m]}=\left[\widehat{\alpha}_{[m]}\{1\}, \widehat{\alpha}_{[m]}\{2\}\right.$, $\left.\ldots, \widehat{\alpha}_{[m]}\{m\}\right] \in \mathfrak{R}^{N+2 m} \times \mathfrak{R}^{m}$. System (17), (20) by virtue of (18) is observable from the available output $y(k)$ for every $\theta$ complying with (19); thus system (17), (20) is expressed in observer canonical form by a suitable coordinate transformation $\zeta\left(\bar{x}_{e}, \bar{w}\right)$

$$
\left\{\begin{array}{l}
\zeta(k+1)=\mathcal{A}_{N+2 m} \zeta(k)-y(k)\left[\widehat{\alpha}_{[m]}\{0\}+\widehat{\alpha}_{[m]} \theta\right] \\
y(k)=\mathcal{C}_{N+2 m} \zeta(k) .
\end{array}\right.
$$

Let $\mathcal{O}_{\bar{x}_{e}} \bar{w}(\theta)$ be the nonsingular observability matrix of system (17), (20) and $\Theta_{\zeta}(\theta)$ be the observability matrix of system (24), that is nonsingular by construction. The transformation from $\left[\bar{x}_{e}, \bar{w}^{T}\right]^{T}$ to $\zeta$ is given by

$\zeta=T_{[m]}(\theta)\left[\begin{array}{c}\bar{x}_{e} \\ \bar{w}\end{array}\right] \quad$ where $T_{[m]}(\theta)=\left[\Theta_{\zeta}^{-1}(\theta) \mathcal{O}_{\bar{x}_{e} \bar{w}}(\theta)\right]$.

Recalling that $\bar{w} \in \mathfrak{R}^{2 m}$ in (20) is bounded by construction, $\zeta(k) \in$ $\mathfrak{R}^{N+2 m}$ is also bounded, since it is diffeomorphic to $\left[\bar{x}_{e}, \bar{w}\right]$ via the nonsingular transformation (25).

Next, we construct an adaptive observer for system (24). Let $d_{[m]} \in \Re^{N+2 m}$ be a vector chosen by the designer such that all the roots of

$p_{d}(z)=z^{N+2 m}+\left[z^{N+2 m-1}, \ldots, z, 1\right] d_{[m]}$

are inside the unit circle; these parameters identify a matrix in observer canonical form defined as $D_{[m]}=\left[\mathcal{A}_{N+2 m}-d_{[m]} \mathcal{C}_{N+2 m}\right]$. Consider the filters

$\xi_{[m]}(k+1)=D_{[m]} \xi_{[m]}(k)-\alpha_{[m]} y(k)$

$\eta_{[m]}(k+1)=D_{[m]} \eta_{[m]}(k)+\left\{d_{[m]}-\widehat{\alpha}_{[m]}\{0\}\right\} y(k)$

with states $\xi_{[m]}(k) \in \mathfrak{R}^{N+2 m}, \eta_{[m]}(k) \in \Re^{N+2 m}$ and arbitrary initial conditions $\xi_{[m]}(0) \in \mathfrak{R}^{N+2 m}, \eta_{[m]}(0) \in \mathfrak{R}^{N+2 m}$. By setting the $m$ vectors $\widehat{\xi}_{[m]}\{i\} \in \Re^{N+2 m}$ as

$\left\{\begin{array}{l}\widehat{\xi}_{[m]}\{i\}=\left[\left(D_{[m]}\right)^{i}+\left(D_{[m]}\right)^{2 m-i}\right] \xi_{[m]}, \\ \quad \text { for all } 1 \leq i \leq m-1 \\ \widehat{\xi}_{[m]}\{m\}=\left(D_{[m]}\right)^{2 m} \xi_{[m]},\end{array}\right.$

and defining the matrix $\widehat{\xi}_{[m]} \in \Re^{N+2 m} \times \Re^{m}$ as $\widehat{\xi}_{[m]}=\left[\widehat{\xi}_{[m]}\{1\}\right.$, $\left.\widehat{\xi}_{[m]}\{2\}, \ldots, \widehat{\xi}_{[m]}\{m\}\right]$, by computing $\widehat{\xi}_{[m]}(k+1)$, by virtue of $(23)$, (27), (29), we have

$\widehat{\xi}_{[m]}(k+1)=D_{[m]} \widehat{\xi}_{[m]}(k)-\widehat{\alpha}_{[m]} y(k)$.

The following property is crucial for the adaptive observer construction of system (24).
Claim 4.1. The vector $\left(\eta_{[m]}(k)+\widehat{\xi}_{[m]}(k) \theta\right) \in \Re^{N+2 m}$ tends exponentially to the state $\zeta(k) \in \Re^{N+2 m}$ of system (24).

Proof. Set

$\Delta_{\zeta}(k)=\zeta(k)-\left(\eta_{[m]}(k)+\widehat{\xi}_{[m]}(k) \theta\right)$.

By computing $\Delta_{\zeta}(k+1)$, by virtue of (31) along with (24), (28), (30) we obtain the dynamics

$\Delta_{\zeta}(k+1)=D_{[m]} \Delta_{\zeta}(k)$

with initial condition $\Delta_{\zeta}(0)=\zeta(0)-\left(\eta_{[m]}(0)+\widehat{\xi}_{[m]}(0) \theta\right)$. The eigenvalues of $D_{[m]}$ are inside the unit circle and (32) implies that $\lim _{k \rightarrow \infty} \Delta_{\zeta}(k)=0$ exponentially.

By multiplying both sides of expression (31) for $\mathcal{C}_{N+2 m}$ and setting

$\bar{y}(k)=y(k)-\mathcal{C}_{N+2} \eta_{[m]}(k)$,

$\mu_{[m]}(k)=\left[\mathcal{C}_{N+2 m} \widehat{\xi}_{[m]}(k)\right]^{T}$,

by rearranging the terms, we obtain an alternative expression for $\bar{y}(k)$, i.e.

$\bar{y}(k)=\mu_{[m]}^{T}(k) \theta+\mathcal{C}_{N+2 m} \Delta_{\zeta}(k)$.

The following property holds:

Claim 4.2. The vector $\mu_{[m]}(k) \in \mathfrak{R}^{m}$ defined in (34) is bounded and persistently exciting, (PE), i.e. there exist an integer $k_{\mu}$ and a positive real $c_{\mu}$ such that

$\sum_{j=k}^{j=k+k_{\mu}} \mu_{[m]}(j) \mu_{[m]}^{T}(j) \geq I_{m} c_{\mu} \quad$ for all $k \geq 0$.

Proof. See Appendix.

We choose a strategy to estimate $\theta \in \mathfrak{R}^{m}$ that leads to exponential convergence via Lyapunov stability theory. To this purpose, consider the scalar function

$S\left(\mu_{[m]}(k)\right)= \begin{cases}c_{1} /\left\|\mu_{[m]}(k)\right\|^{2} & \text { if }\left\|\mu_{[m]}(k)\right\|^{2} \geq \epsilon_{1} \\ c_{1} & \text { if }\left\|\mu_{[m]}(k)\right\|^{2}<\epsilon_{1}\end{cases}$

where $0<c_{1} \leq 1,0<\epsilon_{1} \leq 1$ are tuning parameters chosen by the designer, and set

$\widehat{\theta}_{[m]}(k+1)=\left[I_{m}-S\left(\mu_{[m]}(k)\right) \mu_{[m]}(k) \mu_{[m]}^{T}(k)\right] \widehat{\theta}_{[m]}(k)$

$$
+S\left(\mu_{[m]}(k)\right) \mu_{[m]}(k) \bar{y}(k)
$$

with parameter estimate $\widehat{\theta}_{[m]}(k) \in \Re^{m}$, arbitrary initial condition $\widehat{\theta}_{[m]}(0) \in \mathfrak{R}^{m}$.

Since $\left(\eta_{[m]}(k)+\widehat{\xi}_{[m]}(k) \theta\right) \in \Re^{N+2 m}$ tends exponentially to $\zeta(k) \in \Re^{N+2 m}$, its estimate $\widehat{\zeta}_{[m]}(k)$ is defined accordingly as

$\widehat{\zeta}_{[m]}(k)=\left(\eta_{[m]}(k)+\widehat{\xi}_{[m]}(k) \widehat{\theta}_{[m]}\right)$.

Set $\tilde{\theta}(k)=\theta-\widehat{\theta}_{[m]}(k)$; the computation of $\tilde{\theta}(k+1)$ yields

$\tilde{\theta}(k+1)=\left[I_{m}-\bar{\mu}_{[m]}(k) \bar{\mu}_{[m]}^{T}(k)\right] \tilde{\theta}(k)$

$$
-S\left(\bar{\mu}_{[m]}(k)\right) \mathcal{C}_{N+2 m} \Delta_{\zeta}(k)
$$

where the "normalized" vector $\bar{\mu}(k)$ is defined as

$\bar{\mu}_{[m]}(k)= \begin{cases}c_{1} \mu_{[m]}(k) /\left\|\mu_{[m]}(k)\right\| & \text { if }\left\|\mu_{[m]}(k)\right\|^{2} \geq \epsilon_{1} \\ c_{1} \mu_{[m]}(k) & \text { if }\left\|\mu_{[m]}(k)\right\|^{2}<\epsilon_{1} .\end{cases}$

Then the following property holds:

Claim 4.3. (i) The vectors $\widehat{\theta}_{[m]} \in \Re^{m}$ and $\widehat{\zeta}_{[m]} \in \Re^{N+2 m}$ are globally bounded.(ii) The error estimation vectors $\widetilde{\zeta}=\left[\zeta(k)-\widehat{\zeta}_{[m]}(k)\right] \epsilon$ $\mathfrak{R}^{N+2 m}$ and $\tilde{\theta}(k)=\left[\theta-\widehat{\theta}_{[m]}(k)\right]$ with $\widehat{\theta}_{[m]}(k)$ defined in (38) and $\widehat{\zeta}_{[m]}(k)$ in (39) are bounded exponentially vanishing functions of $k$. 
Proof. See Appendix.

In practical cases the number of excited sinusoids may not be known in advance, so that the construction of an harmonic compensator has to involve a strategy to detect the actual number $m$ excited harmonics.

\subsection{An algorithm to detect the number of excited harmonics}

We present now a strategy to estimate the number $m$ of sinusoidal disturbances, that departs considerably from the approach in [24], describing an algorithm to estimate the sinusoids number in additive white noise. To this purpose, three cascaded filters are introduced: the first one is a filter defined as

$v(k+1)=\left[\mathscr{A}_{2 M+2}-\mathscr{B}_{2 M+2} \bar{a}\right] v(k)+\mathscr{B}_{2 M+2} y(k)$

with state $v=\left[v_{1}, v_{2}, \ldots, v_{2 M+2}\right]^{T} \in \Re^{2 M+2}$, initial condition $v(0) \in \Re^{2 M+2}$, input $y(k)$ given by (17), where the row vector $\bar{a} \in \mathfrak{R}^{2 M+2}$ is a chosen by the designer so that the matrix $\left[\mathcal{A}_{2 M+2}-\mathscr{B}_{2 M+2} \bar{a}\right]$, has its eigenvalues, i.e. the roots of the polynomial

$\bar{p}_{\bar{a}}(z)=\bar{a}\left[1, z, \ldots, z^{2 M+1}\right]^{T}+z^{2 M+2}$,

inside the unit circle. Let $\bar{v}_{i}=\left[v_{1}(k), v_{2}(k), \ldots, v_{2 i}(k)\right] \in \mathfrak{R}^{2 i}$ with $i \in[1, M+1]$ be the vector collecting the first $2 i$ entries of $v \in \mathfrak{R}^{2 M+2}$; it will be shown to be persistently exciting if $1 \leq i \leq m$ and not persistently exciting if $m+1 \leq i \leq M+1$. The vector $v(k) \in \mathfrak{R}^{M+1}$ is the input to the second filter

$$
\left\{\begin{array}{l}
\Omega(k+1)=c_{\Omega} \Omega(k)+v(k) v(k)^{T}, \quad \Omega(0)>0, \\
q_{i}(k)=(10)^{c_{q}(i)}\left|\operatorname{det} \Omega_{i}(k)\right|^{1 / i}, \quad 1 \leq i \leq M+1
\end{array}\right.
$$

with state $\Omega \in \mathfrak{R}^{2 M+2} \times \mathfrak{R}^{2 M+2}, 1 \leq i \leq M+1$, symmetric and positive definite initial condition $\Omega(0)>0$, outputs $q_{i}(k)$, $1 \leq i \leq M+1$, where $\Omega_{i} \in \Re^{i} \times \Re^{i}$ denotes the matrix collecting the first $2 i \times 2 i$ entries of $\Omega$ and $c_{\Omega}, c_{q}(1), \ldots, c_{q}(M+1)$ are scalar design parameters, with $0<c_{\Omega}<1$. Notice that $\Omega$ is symmetric and (44) can be implemented by a filter whose dimension is $2 M^{2}+5 M+3$. It will be shown that the outputs $q_{i}(k)$ with $1 \leq i \leq M+1$ of system (44) comply with the property that

$\int q_{i}(k) \geq q_{M}>0$ for all $k>0$, for all $1 \leq i \leq m$

$\left\{\lim _{k \rightarrow \infty} q_{i}(k)=0, \quad\right.$ exponentially, for all $m+1 \leq i \leq M+1,(45)$

with $q_{M}$ a positive lower bound. The signals $q_{i}(k)$ are the inputs of the third filter

$$
\begin{aligned}
& \chi_{i}(k+1)=\sigma_{i}\left(\chi_{i}(k), q_{i}(k)\right)\left[\chi_{i}(k)+\tilde{\sigma}\left(q_{M+1}\right)\right] ; \\
& \quad \chi_{i}(0)>0 ; 1 \leq i \leq M
\end{aligned}
$$

with state $\chi=\left(\chi_{1}, \ldots, \chi_{M}\right)^{T}$, in which $\tilde{\sigma}\left(q_{M+1}\right)$ and $\sigma_{i}\left(\chi_{i}, q_{i}\right)$, $1 \leq i \leq M$ depend on the tuning parameters $\bar{c} \in \Re^{+}$and $\tilde{c} \in \Re^{+}$along with $\chi_{0} \in \Re^{+}$and are defined as $\tilde{\sigma}\left(q_{M+1}\right)=$ $\left[1-\exp \left(-\tilde{c} q_{M+1}\right)\right]$ and $\sigma_{i}\left(\chi_{i}, q_{i}\right)=\exp \left(-\bar{c} q_{i}\right)$ if $\chi_{i}<\chi_{0}$ while $\sigma_{i}\left(\chi_{i}, q_{i}\right)=\frac{1}{2}$ if $\chi_{i} \geq \chi_{0}$. It is shown in the following that

$\left\{\lim _{k \rightarrow \infty} \chi_{i}(k)=0\right.$ exponentially, for all $1 \leq i \leq m$

$\left\{\begin{array}{l}k \rightarrow \infty \\ \chi_{i}(k) \geq \chi_{M}>0 \text { for all } k>0 \text {, for all } m+1 \leq i \leq M,\end{array}\right.$

where $\chi_{M}$ is a suitable positive lower bound. By virtue of (45), (47) we can derive from the outputs of filters (44) and (46) an estimate $\widehat{m}$ of the integer $m$ by setting

$\widehat{m}(k)=\operatorname{round}\left[\sum_{i=1}^{M} q_{i}(k) /\left[q_{i}(k)+\chi_{i}(k) 10^{\bar{s}}\right]\right]$

where round [ $\cdot$ ] yields the closest integer of its argument and $\bar{s} \in$ $\mathfrak{R}^{+}$is a tuning parameter. The estimation property of the filters introduced so far are stated as follows.
Lemma 4.1. Consider the cascaded interconnection of the filters (42), (44), (46) with input $y(k) \in \Re$ given by (17), state $v(k) \in$ $\mathfrak{R}^{2 M+2}, \Omega(k) \in \mathfrak{R}^{2 M+2} \times \mathfrak{R} 2^{M+2}, \chi(k) \in \mathfrak{R}^{M}$, tuning parameters $0<c_{\Omega}<1, \chi_{0}>0, c_{q} \in \mathfrak{R}^{M+1}$ and $\bar{s} \in \mathfrak{R}^{+}$such that $c_{q}(i)>0$ for all $i \in[1, M+1]$, output $\widehat{m}(k)$ given by (48). The following holds:

(i) the state trajectories are bounded for any $v(0) \in \mathfrak{R}^{2 M+2}, \Omega(0) \in$ $\mathfrak{R}^{2 M+2} \times \mathfrak{R}^{2 M+2}$ such that $\Omega(0)>0$, and any $\chi_{i}(0)>0,1 \leq$ $i \leq M$;

(ii) there exists an integer $k_{m}$ such that for all $k \geq k_{m}$ we have $\widehat{m}(k)=m$.

Proof. see Appendix.

\subsection{Main result}

In this subsection we define an estimate $v_{\delta}(k)$ of the disturbance $\delta(k)$ that will be added in the control law (14) to the stabilizing controller (13), recalling that the number of excited frequencies $m$ is determined online via its estimate $\widehat{m}(k)$. To be specific, consider the filters with states $\eta(k) \in \mathfrak{R}^{N+2 M}, \xi(k) \in \mathfrak{R}^{N+2 M}$

$$
\begin{aligned}
& \eta(k+1)=\left[\begin{array}{cc}
D_{[\widehat{m}]} & 0 \\
0 & c_{0} I_{2 M-2 \widehat{m}}
\end{array}\right] \eta(k)+\left[\begin{array}{c}
d_{[\widehat{m}]}-\alpha_{[\widehat{m}]}\{0\} \\
0
\end{array}\right] y(k) \\
& \xi(k+1)=\left[\begin{array}{cc}
D_{N+2 \widehat{m}} & 0 \\
0 & c_{0} I_{2 M-2 \widehat{m}}
\end{array}\right] \xi(k)-\left[\begin{array}{c}
\alpha_{[\widehat{m}]} \\
0
\end{array}\right] y(k)
\end{aligned}
$$

with arbitrary initial conditions $\eta(0) \in \mathfrak{R}^{N+2 M}, \xi(0) \in \mathfrak{R}^{N+2 M}$, where $0 \leq c_{0}<1$ is a design parameter. The systems above are driven by the output $y(k)$ of system (16), whose dynamics depend on $\widehat{m}(k)$ and $d_{[\widehat{m}]} \in \Re^{N+2 \hat{m}}, D_{[\widehat{m}]} \in \Re^{N+2 \widehat{m}} \times \Re^{N+2 \widehat{m}}$, $\alpha_{[\widehat{m}]}\{0\}, \alpha_{[\widehat{m}]} \in \Re^{N+2 \widehat{m}}$ are defined as in (22) with the estimate $0 \leq \widehat{m}(k) \leq M$ in place of $m$. Consider the first $N+2 \widehat{m}$ entries of $\bar{\eta}(k)$ and $\xi(k)$, i.e. set $\eta_{[\widehat{m}]}(k)=\left[\begin{array}{ll}I_{N+2 \widehat{m}} & 0\end{array}\right] \eta(k)$ and $\xi_{[\widehat{m}]}(k)=$ $\left[\begin{array}{ll}I_{N+2 \widehat{m}} & 0\end{array}\right] \bar{\xi}(k)$. By virtue of Lemma 4.1 there exists an integer $k_{m}$ such that for all $k \geq k_{m}$ we have $\widehat{m}(k)=m$, then the dynamics of $\eta_{[\widehat{m}]}(k)$ and $\xi_{[\widehat{m}]}(k)$ in (49), (50) coincide with the ones of (28), (27) in Section 4 for all $k \geq k_{m}$. If $\widehat{m}(k) \neq 0$ consider the matrix

$\widehat{\xi}_{[\widehat{m}]}=\left[\widehat{\xi}_{[\widehat{m}]}\{1\}, \widehat{\xi}_{[\widehat{m}]}\{2\}, \ldots, \widehat{\xi}_{[\widehat{m}]}\{\widehat{m}\}\right] \in \mathfrak{R}^{N+2 \widehat{m}} \times \mathfrak{R}^{\widehat{m}}$,

whose columns $\widehat{\xi}_{[\widehat{m}]}\{1\}, \ldots, \widehat{\xi}_{[\widehat{m}]}\{\widehat{m}\}$ are defined as in (29) with $1 \leq \widehat{m}(k) \leq M$ in place of $m$. In accordance with (33), (34) set $\bar{y}(k)=y(k)-C_{N+2 \widehat{m}} \eta_{[\widehat{m}]}(k)$ and $\mu_{[\widehat{m}]}(k)=\left[\mathcal{C}_{N+2 \widehat{m} \widehat{\xi}_{[\widehat{m}]}}(k)\right]^{T}$. Consider the system with state $\widehat{\theta}(k) \in \mathfrak{R}^{M}$, described by

$$
\begin{aligned}
\widehat{\theta}(k+1)= & {\left[\begin{array}{cc}
\left(I_{\widehat{m}}-S\left(\mu_{[\widehat{m}]}(k)\right) \mu_{[\widehat{m}]}(k) \mu_{[\widehat{m}]}^{T}(k)\right) & 0 \\
0 & c_{0} I_{M-\widehat{m}}
\end{array}\right] \widehat{\theta}(k) } \\
& +\left[\begin{array}{c}
S\left(\mu_{[\widehat{m}]}(k)\right) \mu_{[\widehat{m}]}(k) \\
0
\end{array}\right] \bar{y}(k)
\end{aligned}
$$

arbitrary initial condition $\widehat{\theta}(0) \in \mathfrak{R}^{M}$, if $\widehat{m}(k) \neq 0$, while $\widehat{\theta}(k+1)=$ 0 if $\widehat{m}(k)=0$. The estimation algorithm described so far, satisfies the following properties.

Claim 4.4. (i) The vectors $\eta(k) \in \mathfrak{R}^{N+2 M}, \xi(k) \in \mathfrak{R}^{N+2 M}, \widehat{\theta}(k) \in$ $\mathfrak{R}^{M}$ and are globally bounded for all $k>0$.

(ii) The vectors $\hat{\theta}_{[\widehat{m}]}(k)=\left[\begin{array}{ll}I_{\widehat{m}} & 0\end{array}\right] \widehat{\theta}(k)$ and $\widehat{\zeta}_{[\widehat{m}]}(k)=\left(\eta_{[\widehat{m}]}(k)+\right.$ $\left.\widehat{\xi}_{[\widehat{m}]}(k) \widehat{\theta}_{[\widehat{m}]}\right)$, are exponentially convergent estimates of $\theta \in \Re^{m}$ and $\zeta \in \mathfrak{R}^{N+2 m}$, respectively.

(iii) the last $2(M-\widehat{m})$ entries of $\eta(k) \in \mathfrak{R}^{N+2 M}$ and $\xi(k) \in \mathfrak{R}^{N+2 M}$ tend exponentially to zero, as well as the last $M-m$ entries of $\widehat{\theta}(k) \in \mathfrak{R}^{M}$.

Proof. The results are a consequence of Claim 4.3 along with Lemma 4.1. 


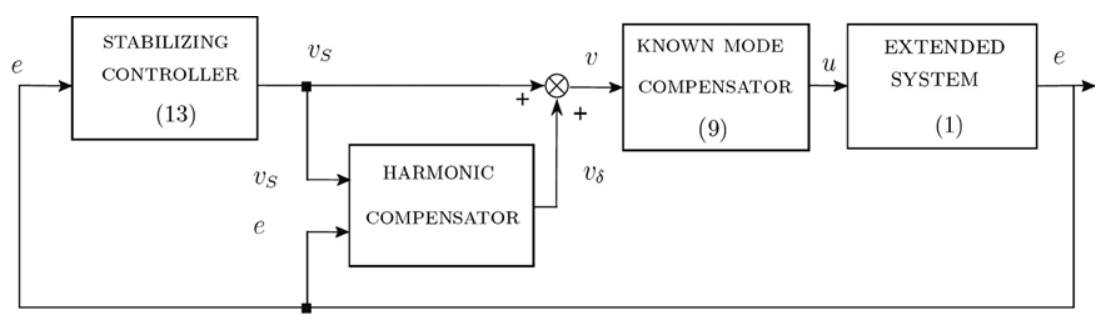

Fig. 1. Adaptive regulator in Problem 2.1.

Recall that $\delta(k)$ is the first entry of the vector $\bar{w} \in \mathfrak{R}^{2 m}$, which is related to $\zeta$ via the transformation $\left[\bar{x}_{e}^{T} \bar{w}^{T}\right]^{T}=T_{[m]}^{-1}(\theta) \zeta$. By setting $\rho_{[m]}(k)=r_{[m]}(\theta) \zeta(k)$, where $r_{[m]}(\theta)$ is the $(N+1)$ th row of adjoint $\left[T_{[m]}(\theta)\right], \delta(k)$ it is expressed as

$\delta(k)=\frac{\rho_{[m]}(k)}{\operatorname{det} T_{[m]}(\theta)}$.

Let $r_{[\widehat{m}]}\left(\widehat{\theta}_{[\widehat{m}]}(k)\right)$ be the $(N+1)$ th row of the adjoint of the matrix $T_{[\widehat{m}]}\left(\widehat{\theta}_{[\widehat{m}]}(k)\right)$ which is defined as in (25) with $\widehat{m}(k)$ in place of $m$. Set

$\widehat{\rho}_{[\widehat{m}]}(k)=r_{[\widehat{m}]}\left(\widehat{\theta}_{[\widehat{m}]}(k)\right) \widehat{\zeta}_{[\widehat{m}]}(k)$.

By Claim 4.4, $\left.\widehat{\rho}_{[\widehat{m}]}(k) / T_{[\widehat{m}]} \widehat{\theta}_{[\widehat{m}]}(k)\right)$ tends exponentially to $\delta(k)$, although is not guaranteed to be bounded in its transient performance. To avoid the singularities in which $\operatorname{det} T_{[\widehat{m}]}\left(\widehat{\theta}_{[\widehat{m}]}(k)\right)=0$, consider the adaptive saturation algorithm

$$
\left\{\begin{array}{l}
x_{p}(k+1)=c_{2} x_{p}(k)+G(k), \quad x_{p}(0)>0 \\
p(k)=c_{3}\left(x_{p}(k)+G(k)\right)
\end{array}\right.
$$

with state $x_{p}(k) \in \mathfrak{R}$, any initial condition such that $x_{p}(0)>0$ and $c_{2}, c_{3}$ positive design parameters with $c_{2}<1$, driven by the scalar function

$G(k)=\frac{2}{\pi} \arctan \left(c_{4}\|\widehat{\theta}(k+1)-\widehat{\theta}(k)\|\right)$

where $c_{4}$ is a tuning parameter. Since system (54) is driven by $G(k)$ which tends exponentially to zero, then $p(k)>0$ and $\lim _{k \rightarrow \infty}$ $p(k)=0$ exponentially. The functions $p(k) \in \Re^{+}, \widehat{\rho}_{[\widehat{m}]}(k) \in \mathfrak{R}$ in (53) and det $T_{[m]}\left(\widehat{\theta}_{[m]}(k)\right) \in \Re$ provide the estimate $v_{\delta}(k)$ of $\delta(k)$ via the adaptive saturation algorithm

$v_{\delta}(k)=\left\{\begin{array}{l}0 \quad \text { if } \widehat{m}(k)=0 \\ {\left[\widehat{\rho}_{[\widehat{m}]]}(k) \operatorname{det} T_{[\widehat{m}]}\left(\widehat{\theta}_{[\widehat{m}]}(k)\right)\right] / p^{2}(k)} \\ \quad \text { if } \widehat{m}(k) \neq 0 \text { and }\left|\operatorname{det} T_{[\widehat{m}]}\left(\widehat{\theta}_{[\widehat{m}]}(k)\right)\right| \leq p(k) \\ \widehat{\rho}_{[\widehat{m}]]}(k) / \operatorname{det} T_{[\widehat{m}]}\left(\widehat{\theta}_{[\widehat{m}]}(k)\right) \\ \quad \text { if } \widehat{m}(k) \neq 0 \text { and }\left|\operatorname{det} T_{[\widehat{m}]}\left(\widehat{\theta}_{[\widehat{m}]}(k)\right)\right|>p(k) .\end{array}\right.$

The harmonic compensator described so far, shown in Fig. 2, along with the stabilizing controller (9), (13), (14), solves Problem 2.1. We state now the main result of this paper.

Proposition 4.1. Consider system (1). Assume that hypotheses (H1)-(H3) hold. Then Problem 2.1 is solved via the dynamic output feedback compensator (14), (56), with dynamics (9), (13), (16), (42), (44), (46), (49), (50), (51), (54), whose state is $s \in \Re^{r_{1}}, X \in \Re^{n_{1}}, \widehat{x}_{e} \in$ $\mathfrak{R}^{N}, v \in \Re^{2 M+2}, \Omega \in \mathfrak{R}^{2 M+2} \times \mathfrak{R}^{2 M+2}, \chi \in \mathfrak{R}^{M}, \eta \in \mathfrak{R}^{N+2 M}$, $\xi \in \mathfrak{R}^{N+2 M}, \widehat{\theta} \in \mathfrak{R}^{M}, x_{p} \in \mathfrak{R}$. In particular, the closed loop system is such that: its trajectories are bounded and the output $e(k)$ tends exponentially to zero as $k$ goes to infinity for any initial condition $x(0) \in \Re^{n}, w(0) \in \Re^{r}$, of system (1) and any initial condition of the regulator such that $\Omega(0)>0, \chi_{i}(0)>0,1 \leq i \leq M, x_{p}(0)>0$.

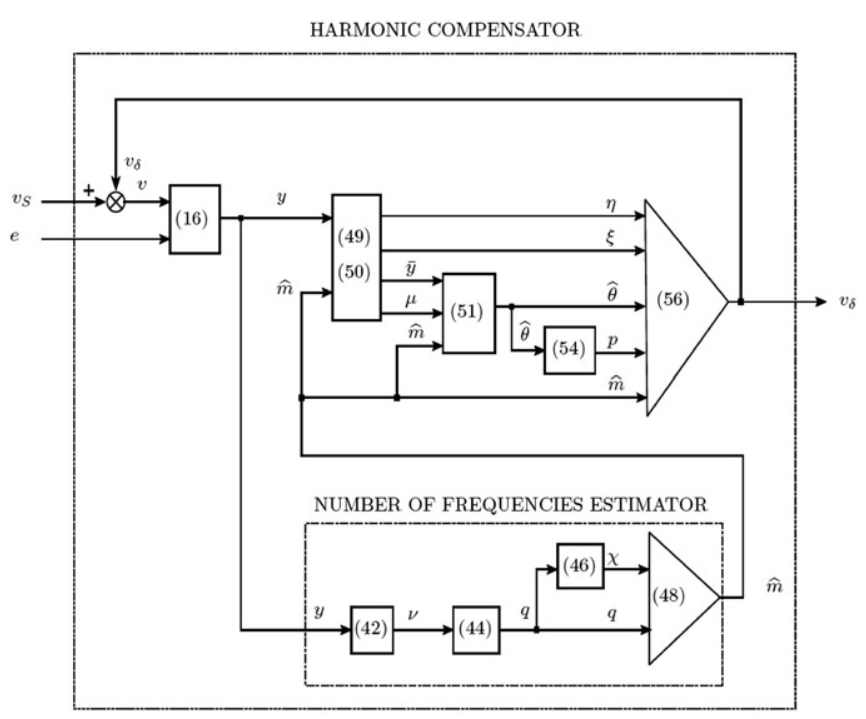

Fig. 2. The harmonic compensator developed in Section 4, as a part of the regulator in Fig. 1.

Proof. By Claim 4.4 the vectors $\eta_{[m]} \in \mathfrak{R}^{N+2 m}, \xi_{[\widehat{m}]} \in \mathfrak{R}^{N+2 \widehat{m}}$, $\widehat{\theta}_{[\widehat{m}]} \in \mathfrak{R}^{\widehat{m}}$ and $\widehat{\zeta}_{[\widehat{m}]} \in \mathfrak{R}^{N+2 \widehat{m}}$ are globally bounded, hence also the function $\widehat{\rho}_{[\widehat{m}]}(k)$ is bounded, and we have $\left|\widehat{\rho}_{[\widehat{m}]}(k)\right| \leq \rho_{M}$ for all $k \geq 0$, where $\rho_{M}$ is a suitable positive real. Next, we show that the disturbance estimation error $\delta(k)-v_{\delta}(k)$ is globally exponentially vanishing. From (56) we have

$\left|v_{\delta}(k)\right| \leq\left|\widehat{\rho}_{[\widehat{m}]}(k)\right| /|p(k)| \quad$ for all $k \geq 0$.

From (54) recalling that the function $G(k)$ is exponentially vanishing by virtue of Claim 4.3 , we deduce that $p(0) c_{2}^{k} \leq p(k) \leq$ $c_{p} \lambda_{p}^{k}$ for all $k \geq 0$, and suitable positive real numbers $c_{p}$ and $\lambda_{p}$ with $\lambda_{p}<1$. Since by Claim 4.4 the vector $\left(\theta-\widehat{\theta}_{[\widehat{m}]}(k)\right)$ is exponentially vanishing, then

$\left.\mid \operatorname{det} T_{[\widehat{m}]} \widehat{\theta}_{[\widehat{m}]}(k)\right)|\geq| \operatorname{det} T_{[m]}(\theta) \mid-c_{T} \lambda_{s}^{k}$

for suitable positive real numbers $c_{T}$ and $\lambda_{s}$ with $\lambda_{s}<1$. Let $\bar{c}$ $=\max \left\{c_{T}, c_{p}\right\}$ and $\bar{\lambda}=\min \left\{\lambda_{s}, \lambda_{p}\right\}$; from (58), recalling that $p(k) \leq c_{p} \lambda_{p}^{k}$, we have that

$p(k) \leq \bar{c} \bar{\lambda}^{k}$ and $\left.\mid \operatorname{det} T_{[\widehat{m}]} \widehat{\theta}_{[\widehat{m}]}(k)\right)|\geq| \operatorname{det} T_{[m]}(\theta) \mid-\bar{c} \bar{\lambda}^{k}$

for all $k>0$.

By setting $\bar{k}=\log _{\bar{\lambda}}\left[\left|\operatorname{det} T_{[m]}(\theta)\right| / 2 \bar{c}\right]$, from (59) we deduce that $\left|\operatorname{det} T_{[\hat{m}]}\left(\widehat{\theta}_{[\widehat{m}]}(k)\right)\right|>p(k)$ for all $k>\bar{k}$, so that by virtue of (54) $v_{\delta}(k)=\widehat{\rho}_{[\hat{m}]}(k) /\left|\operatorname{det} T_{[\widehat{m}]}\left(\widehat{\theta}_{[\widehat{m}]}(k)\right)\right|$ for all $k>\bar{k}$ and $\left|\delta(k)-v_{\delta}(k)\right|$ $\leq \bar{c}_{\delta} \lambda_{\delta}^{k}$ if $k>\bar{k}$ for suitable positive real numbers $\bar{c}_{\delta}$ and $\lambda_{\delta}$ with $\lambda_{\delta}<1$. Notice that for $0 \leq k \leq \bar{k}$ the function $\left|\delta(k)-v_{\delta}(k)\right|$ is bounded; in fact from (57) recalling that $\left|\widehat{\rho}_{[\widehat{m}]}(k)\right| \leq \rho_{M}$, we have $\sup _{0 \leq k \leq \bar{k}}\left|\delta(k)-v_{\delta}(k)\right| \leq \sup _{0 \leq k \leq \bar{k}}|\delta(k)|+\frac{\rho_{M}}{p(0)} c_{2}^{-\bar{k}}$. We conclude 

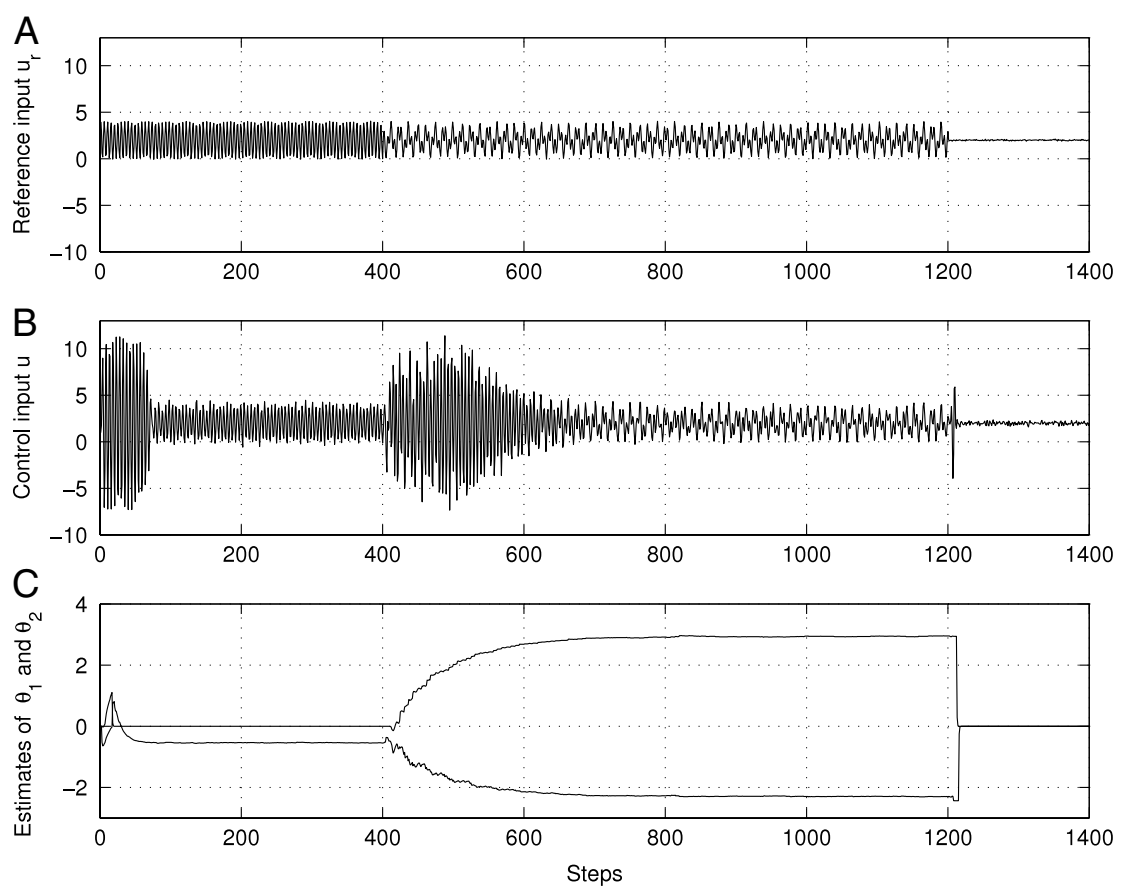

Fig. 3. (A) The reference input $u_{r}(k)$; (B) the control input $u(k)$; (C) the exosystem parameter estimates $\widehat{\theta}_{1}(k), \widehat{\theta}_{2}(k)$.

that $\left|\delta(k)-v_{\delta}(k)\right| \leq c_{\delta} \lambda_{\delta}^{k}$ for all $k \geq 0$ and $c_{\delta}=\lambda_{\delta}^{-\bar{k}} \max \left\{\sup _{0 \leq k \leq \bar{k}}\right.$ $\left.\left|\delta(k)-v_{\delta}(k)\right|, \bar{c}_{\delta}\right\}$. By setting $\bar{X}=\left[x_{e}^{T}, X^{T}, \bar{w}^{T}\right]^{T}$, the closed loop system (11), (13), (20), by virtue of (14), becomes $\bar{X}(k+1)=$ $\bar{A} \bar{X}+\bar{b}\left(\delta(k)-v_{\delta}(k)\right)$, where $\bar{b}$ is a constant vector and $\bar{A}$ is a Hurwitz matrix by construction. Recalling that $\left|\delta(k)-v_{\delta}(k)\right| \leq$ $c_{\delta} \lambda_{\delta}^{k}$ we conclude that the vector $\bar{X}(k)$ and the output $e(k)$ are bounded and globally exponentially vanishing. The state $x_{u}(k) \in$ $\Re^{n-n_{0}}$ of system (4) is also bounded and globally exponentially vanishing since the matrix $A_{u}$ in (4) has the eigenvalues inside the unit circle and both $x_{0}(k)$ and $u(k)-u_{r}(k)$ are bounded and globally exponentially vanishing.

\section{An example}

Consider a system in the form (3), described by the set of differential equations

$\left\{\begin{array}{l}x(k+1)=\left[\begin{array}{cc}0.3 & 1 \\ 1 & 0\end{array}\right] x(k)+\left[\begin{array}{l}1 \\ 2\end{array}\right]\left(u(k)-u_{r}(k)\right) \\ e(k)=\left[\begin{array}{ll}1 & 0\end{array}\right] x(k),\end{array}\right.$

with state $x=\left[x_{1}, x_{2}\right]^{T} \in \Re^{2}$, control input $u \in \Re$, output $e \in \Re$ to be regulated to zero, reference input $u_{r} \in \Re$. System (60) has unstable unforced dynamics and is non-minimum phase. We set $u_{r}(k)=2+\bar{\delta}(k)+v(k)$, where $\bar{\delta}(k)$ is an harmonic disturbance and $v(k)$ is an unmodelled noise produced with the MatLab Simulink Block 'band limited white noise' with power equal to 0.002 and initial seed equal to zero. To compensate the unknown bias equal to 2 , we define a known mode compensator (9) setting $s(k+1)=s(k)+v(k)$ and $u(k)=s(k)$ that yields along with (60) a system in the form (11) perturbed by $\delta(k)=$ $\bar{\delta}(k+1)-\bar{\delta}(k)$. We consider a stabilizing controller in the form (13), starting from zero initial conditions, whose transfer function from $e(k)$ to $v_{S}(k)$ is $W_{S}=\frac{0.2 z^{2}+2.8 z+2.36}{z^{3}+2.3 z^{2}+3.24 z+1.94}$. This is a robust discrete $H^{\infty}$ dynamic controller produced via the MatLab function dhinf (see MatLab 5.3 Reference Manual). We assume to have three operating conditions for $\bar{\delta}(k)$ (see Box I).

We set the harmonic upper bound $M=2$, and simulate the algorithm for $0<k \leq 1400$ choosing the numerical values of the constant design parameters as follows: in system (16) we choose $a_{1}, a_{2}, a_{3}$, so that all the eigenvalues of $p_{a}(z)$ in (15) coincide with 0.2 . In system (42) we choose a vector $\bar{a}$ associated with a polynomial (43) whose roots coincide with the number 0.2 . In system (44) we let $c_{\Omega}=0.4$ and $c_{q}=[3.6 ; 3.6 ; 8]$. In system (46) we set $\chi_{0}=1, \bar{c}=10$, and $\tilde{c}=1$. In expression (48) we set $\bar{s}=6.5$. In (49), (50) we consider the vectors $d_{[1]} d_{[2]}$, that are associated to polynomials $p_{d}(z)$ in (26) with eigenvalues in 0.2 and set $c_{0}=0.1$. The parameters of the function $S\left(\mu_{[\widehat{m}]}(k)\right)$ defined in (37) are $\epsilon_{1}=1, c_{1}=0.1$. In system (54) we set $c_{2}=0.9, c_{3}=10$ and in (55) $c_{4}=50$. The compensator dynamics have been simulated starting from zero initial conditions except for $\Omega(0)=I_{6}, \chi_{i}(0)=1, i \in[1,2,3]$ and $x_{p}(0)=100$.

The simulation results are reported in Figs. 3, 4. In Fig. 3 the reference input $u_{r}(k)$, the control input $u(k)$, and the estimates $\widehat{\theta}_{1}(k), \widehat{\theta}_{2}(k)$, of the parameters $\theta_{1}, \theta_{2}$, are displayed. It illustrates that the three operating conditions involving a different minimal excited harmonics exosystem structure are detected by the observation algorithm. The upper plot (A) in Fig. 4 reports the output $e(k)$ in the setting considered so far; the central plot (B) in Fig. 4 displays $|e(k)|_{d B}$, i.e. its absolute value expressed in decibel. The lower plot $(\mathrm{C})$ in Fig. 4 reports $|e(k)|_{d B}$ in a different setting: when $u_{r}(k)=\bar{\delta}(k)$ (that is in absence of noise). The plot in Fig. 4(C) illustrates that without unmodelled noise $v(k)$ the regulation strategy may drive exponentially to zero the output $e(k)$.

\section{Conclusion}

In this paper we have solved the problem of exponentially driving to zero the output regulation error of a known, discrete time, linear system allowed to be non-minimum phase (NMP), whose disturbances and/or references are generated by an unknown exosystem with simple eigenvalues on the unit circle, under the minimal set of hypotheses for the problem to admit a solution. The exponential convergence results are guaranteed from any initial condition of both the regulated system and the exosystem, i.e. even when the initial conditions excite a subset, possibly empty, of the exosystem modes. The algorithm includes a module to detect the number of the unknown excited frequencies and provides exponentially convergent estimates of the unknown 


\begin{tabular}{|c|c|c|}
\hline (i) & (ii) & (iii) \\
\hline Time: $t \leq 400$ & Time: $400<k \leq 1200$ & Time: $1200<t$ \\
\hline Harmonic Input $:$ & Harmonic Input : & Harmonic Input : \\
$\bar{\delta}(k)=\sin (1.3 k)$ & $\bar{\delta}(k)=\sin (1.3 k)-\sin (0.5 k)=0$ \\
\hline Minimal Exosystem $(20):$ & Minimal Exosystem $(20):$ & \\
$\left\{\begin{array}{l}\dot{\bar{w}}_{1}=-0.534 \bar{w}_{1}+\bar{w}_{2} \\
\dot{\bar{w}}_{2}=\bar{w}_{1}\end{array}\right.$ & $\left\{\begin{array}{l}\overline{\bar{w}}_{1}=-2.290 \bar{w}_{1}+\bar{w}_{2} \\
\overline{\bar{w}}_{2}=2.939 \bar{w}_{1}+\bar{w}_{3} \\
\dot{\bar{w}}_{3}=-2.290 \bar{w}_{1}+\bar{w}_{4} \\
\overline{\bar{w}}_{4}=\bar{w}_{1}\end{array}\right.$ & No Excited Harmonics \\
\hline
\end{tabular}

Box I.
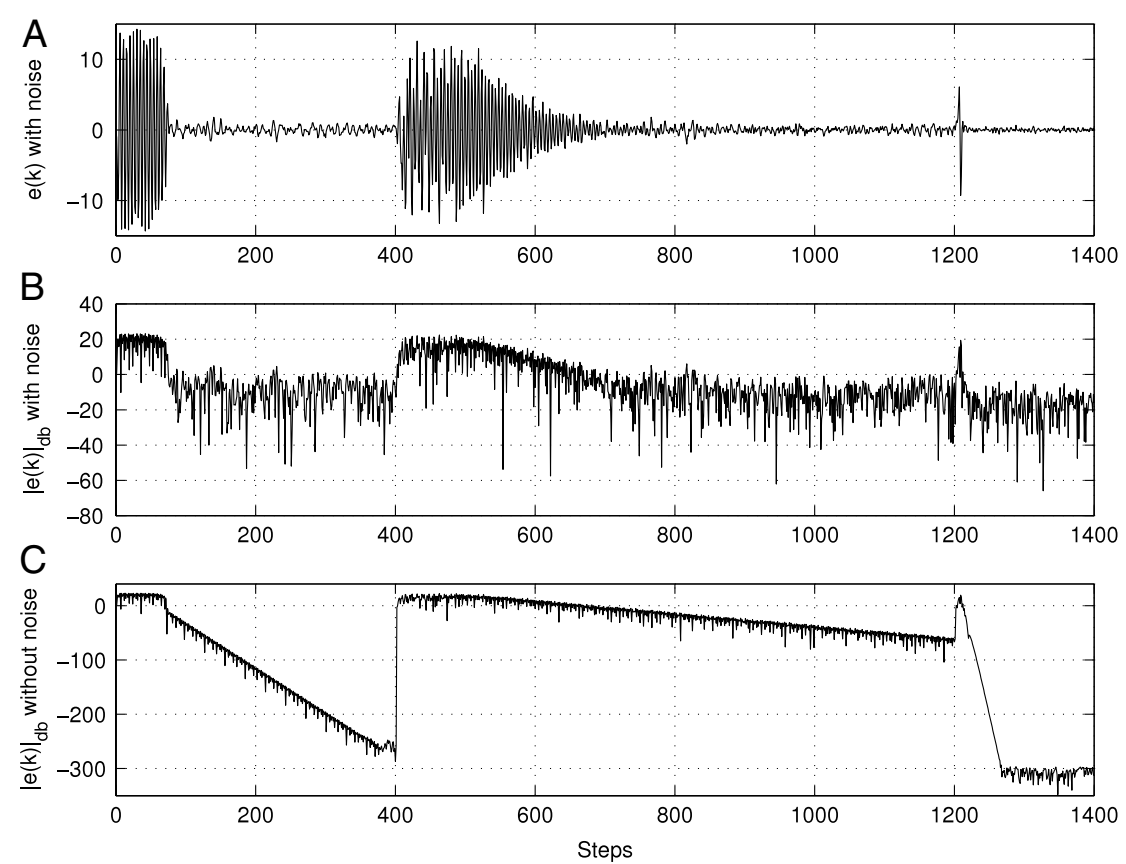

Fig. 4. (A) The regulation error $e(k)$ with exact regulated system parameters and noisy disturbance; (B) $|e(k)|_{d B}$ in the same setting; (C) $|e(k)|_{d B}$ without noise.

exosystem parameters for any initial condition of the estimates. The controller is tested on a numerical example in the presence of a disturbance affecting the input channel: the disturbance is chosen to be a biased sum of sinusoids with piecewise constant frequencies and abrupt changes in their number and frequencies, showing the capability of the algorithm to adapt to a varying disturbance and its robustness with respect to noises. The simulation performed on a numerical example achieves exact exponential regulation for a purely harmonic input reference.

Notice that the methodology described here may inspire other algorithms to solve also a different problem with respect to the one considered in this paper, i.e. the case in which harmonic disturbances affect the output channel. However, its implementation for NMP systems requires a specific strategy which will be the object of future research.

\section{Appendix}

Proof of Claim 4.2. In what follows we will set $\mu=\mu_{[m]}$ and $\bar{\mu}=\bar{\mu}_{[m]}$ for conciseness. First, recall that the vector $\bar{\xi}_{[m]}$ and the entries of the matrix $\widehat{\xi}_{[m]} \in \mathfrak{R}^{N+2 m} \times \mathfrak{R}^{m}$ are bounded, being linear combinations of the entries of the bounded vector $\xi_{[\mathrm{m}]}$; so that $\mu=\bar{\mu}_{[m]}=\mathcal{C}_{v+2 m} \widehat{\xi}_{[m]}$ is also bounded. If PE holds, given any constant vector $\varrho=\left[\varrho_{1}, \varrho_{2}, \ldots, \varrho_{m}\right]^{T}$ with $\|\varrho\|=1$, by computing the expression $\varrho^{T}\left[\sum_{j=k}^{j=k+k_{\mu}} \mu(j) \mu^{T}(j)\right] \varrho$ and setting $Y_{\varrho}(k)=\mu^{T}(k) \varrho$, (36) yields $\sum_{j=k}^{j=k+k_{\mu}} Y_{\varrho}^{2}(j) \geq c_{\mu}$ for all $k \geq 0$.
By contradiction, assume that PE does not hold; then there exists a constant vector $\varrho \in \mathfrak{R}^{m}$ such that we have $\lim _{k \rightarrow \infty} Y_{\varrho}(k)=0$. Let $p_{\rho}(z)=\varrho_{1} z^{2 m-1}+\cdots+\varrho_{m-1} z^{m+1}+\varrho_{m} z^{m}+\varrho_{m-1} z^{m-1}+$ $\cdots+\varrho_{1} z$; the scalar function $Y_{\varrho}(k)$ can be shown to be the output of the linear filter with input $y(k)$ and transfer function $W_{\varrho}(z)=$ $\left(p_{a}(z) p_{\rho}(z)\right) / p_{d}(z)$. The signal $y(k)$ is the output of system (17) with input $\bar{\delta}(k)$ and transfer function given by (18). By setting $\mathcal{Z}[\bar{\delta}(k)]=N_{\delta}(z) / p_{\delta}(z)$ with $p_{\delta}(z)$ is defined in (19) and $N_{\delta}(z)$ suitable polynomial, then $\mathcal{Z}\left[Y_{\varrho}(k)\right]=-\frac{p_{b}(z) p_{\rho}(z)}{p_{d}(z)} \frac{N_{\delta}(z)}{p_{\delta}(z)}+\frac{N_{2}(z)}{p_{d}(z) p_{a}(z)}$, where $N_{2}(z)$ is a suitable polynomial. The last equality implies that in $\mathcal{Z}\left[Y_{\varrho}(k)\right]$ there is at least the $z$-transform of a sinusoid, contradicting that $\lim _{k \rightarrow \infty} Y_{\varrho}(k)=0$.

Lemma A.1. Consider the nonlinear system $x(k+1)=f(x(k), k)+$ $g(x(k), k) u(k)$, with state $x(k) \in \Re^{n}$ and input $u(k) \in \Re^{m}$. Let $\|f(x(k), k)\| \leq c_{f}\|x(k)\|$ and $\|g(x(k), k)\| \leq c_{g}$ for all $x(k) \in \Re^{n}$ and $k \geq 0$, with $c_{f}$ and $c_{g}$ positive real numbers. Assume that there exists a Lyapunov function $V(x, k)$ and positive numbers $\alpha_{i} \in \mathfrak{R}^{+}$, $i \leq i \leq 4$ such that:

(i) $\alpha_{1}\|x\|^{2} \leq V(x, k) \leq \alpha_{2}\|x\|^{2}$ for all $x \in \mathfrak{R}^{n}$ and $k \geq 0$.

(ii) $V\left(x_{a}+\bar{x}_{b}, k\right) \leq \bar{V}\left(x_{a}, k\right)+V\left(x_{b}, k\right)+\alpha_{3}\left\|x_{a}\right\|\left\|x_{b}\right\|$ for all $x_{a} \in \mathfrak{R}^{n}, x_{b} \in \mathfrak{R}^{n}$, and $k \geq 0$.

(iii) $V(f(x(k), k), k+1)-V(x(k)) \leq-\alpha_{4}\|x(k)\|^{2}$.

If $u(k)$ is a bounded and globally exponentially vanishing function of $k$, then $x(k)$ is also a bounded and globally exponentially vanishing function of $k$. 
Proof. It is straightforward via standard Lyapunov techniques.

Proof of Claim 4.3. We show that system (40) complies with Lemma A. 1 with $\widetilde{\theta}$ in place of $x$ and $\Delta_{\zeta}$ in place of $u$. In fact by Claim 4.3, we infer that the preliminary properties of Lemma A.1 are satisfied. Consider now the Lyapunov function $V_{1}(\widetilde{\theta}, k)=$ $\widetilde{\theta}^{T}\left(I_{m}+\frac{2}{5} Q(k)\right) \widetilde{\theta}$, where $Q(k) \in \Re^{m} \times \Re^{m}$ is the state of the filter with dynamics $Q(k+1)=\frac{1}{2}\left(Q(k)+\bar{\mu}(k) \bar{\mu}^{T}(k)\right)$, initial state $Q(0) \in \mathfrak{R}^{m} \times \Re^{m}$ such that $\|Q(0)\|<1$. It can be shown by induction that there exists a suitable positive real $q_{m}<1$, such that $q_{m} I_{m} \leq Q(k) \leq I_{m}$ for all $k>0$. From the last inequalities we infer that properties (i) and (ii) of Lemma A.1 hold for $V_{1}(\widetilde{\theta}, k)$. In order to show property (iii), set $V_{1}(k) \triangleq\left\|\widetilde{\theta}^{2}\right\|+\frac{2}{5} \tilde{\theta}^{T} Q(k) \widetilde{\theta}$ and $V_{1}(k+1) \triangleq$ $V_{1}\left(\left[I_{m}-\bar{\mu} \bar{\mu}^{T}\right] \widetilde{\theta}, k+1\right)$ where for conciseness $\widetilde{\theta}=\tilde{\theta}(k), \bar{\mu}=$ $\bar{\mu}(k)$. By completing the squares, straightforward computations lead to the inequality $V_{1}(k+1)-V_{1}(k) \leq-\left(\tilde{\theta}^{T} Q(k) \widetilde{\theta}\right) / 10 \leq$ $-q_{m}\left\|\widetilde{\theta}^{2}\right\| / 10$, which is a particularization of (iii) in Lemma A.1 with $q_{m} / 10$ in place of $\alpha_{4}$.

Proof of Lemma 4.1. Let $\bar{v}_{i}(k)$ denote the first $2 i$ entries of $v \in$ $\Re^{2 M+2}$; for any vector $\bar{b}^{[i]}=\left[\bar{b}_{1}, \bar{b}_{2}, \ldots, \bar{b}_{2 i}\right] \in \mathfrak{R}^{2 i}$, we have $\bar{b}^{[i]} \bar{v}_{i}(k)=\epsilon(k)+\mathfrak{Z}^{-1}\left[\frac{\left(\bar{b}_{1}+\bar{b}_{2} z+\cdots+\bar{b}_{2 i} z^{2 i-1}\right) p_{b}(z) N_{\delta}(z)}{\bar{p}_{\bar{a}}(z) p_{a}(z) p_{\delta}(z)}\right]$, where $\epsilon(k)$ is an exponentially vanishing function, $N_{\delta}(z)$ is a suitable polynomial and $\bar{p}_{\bar{a}}(z), p_{a}(z), p_{\delta}(z)$ defined in (43), (15), (19) respectively. If $m+1 \leq i \leq M+1$, by setting $\bar{b}^{[i]}=\left[1, \theta_{1}, \ldots\right.$ $\left.\theta_{m-1}, \theta_{m}, \theta_{m-1}, \theta_{1}, 1,0, \ldots, 0\right] \in \mathfrak{R}^{2 i}$, we obtain $\lim _{k \rightarrow \infty} \bar{b}^{[i]} \bar{\nu}_{i}(k)$ $=0$ and this implies that $\lim _{k \rightarrow \infty} q_{i}(k)=0$ exponentially. To the contrary, if $i \leq m$, it does not exist a vector $\bar{b}^{[i]} \in \mathfrak{R}^{2 i}$ such that $\lim _{k \rightarrow \infty} \bar{v}_{i}(k) \overline{\bar{b}}^{[i]}=0$ and we conclude that (45) holds. Consider now system (46): if $1 \leq i \leq m$ by virtue of (45), recalling that $q_{M+1}(k)$ is exponentially vanishing we deduce that $\lim _{k \rightarrow \infty}$ $\chi_{i}(k)=0$ exponentially. If $m+1 \leq i \leq M$ since $q_{i}(k)$ is exponentially vanishing then $q_{i}(k) \leq q_{M} \lambda_{q}^{k}$ for suitable positive real numbers $q_{M}, \lambda_{q}<1$ and $1 \geq \sigma_{i}\left(q_{i}(k)\right) \geq \exp \left(-q_{M} \lambda_{q}^{k}\right)$; from (46) we infer that $\chi_{i}(k) \geq \chi_{M}=\chi_{0} \exp \left(-\frac{q_{M} \lambda_{q}}{1-\lambda_{q}}\right)>0$ for all $k$ $\geq 0$ and we conclude that (47) holds. The convergence properties of the harmonics number estimate $\widehat{m}(k)$ in (48) is a consequence of (45) and (47), the asymptotic properties of $q_{i}(k)$ and $\chi_{i}(k)$, for $1 \leq i \leq M$.

\section{References}

[1] B.A. Francis, W.M. Wonham, The internal model principle of control theory, Automatica 12 (5) (1976) 457-465.
[2] I.D. Landau, A. Constantinescu, D. Rey, Adaptive narrow band disturbance rejection applied to an active suspension-an internal model principle approach, Automatica 41 (4) (2005) 563-574.

[3] A. Saks, M. Bodson, P. Khosla, Experimental results of adaptive periodic disturbance cancellation in a high performance magnetic disk drive, ASME J. Dynam. Syst. Meas. Control 118 (3) (1996) 416-424.

[4] C.C. De Wit, L. Praly, Adaptive eccentricity compensation, IEEE Trans. Control Syst. Technol. 8 (5) (2000) 757-766.

[5] S. Kuo, D. Morgan, Active Noise Control Systems: Algorithms and DSP Implementations, Wiley, New York, NY, 1996.

[6] V.A. Blasko, A novel method for selective harmonic elimination in power electronic equipment, IEEE Trans. Power Electron. 22 (1) (2007) 223-228.

[7] S. Bittanti, L. Moiraghi, Active control of vibrations in helicopters via pole assignment techniques, IEEE Trans. Control Syst. Technol. 2 (4) (1994) 343-350.

[8] K.B. Ariyur, M. Krstic, Feedback attenuation and adaptive cancellation of blade vortex interaction on a helicopter blade element, IEEE Trans. Control Syst. Technol. 7 (5) (1999) 596-605.

[9] G.C. Goodwin, S. Chan, Model reference adaptive control of systems having purely deterministic disturbances, IEEE Trans. Automat. Control 28 (8) (1983) 855-858.

[10] G.C. Goodwin, P.J. Ramadge, P. Caines, Discrete time multivariable adaptive control, IEEE Trans. Automat. Control 25 (3) (1980) 449-456.

[11] F. Ben Amara, P. Kabamba, A. Ulsoy, Adaptive band-limited disturbance rejection in linear discrete-time systems, Math. Probl. Eng. 1 (1995) 139-177.

[12] F. Ben Amara, P. Kabamba, A. Ulsoy, Adaptive sinusoidal disturbance rejection in linear discrete-time systems-part I: theory, J. Dyn. Syst. Meas. Control 121 (1999) 648-654.

[13] M. Ficoncelli, F. Ben Amara, Adaptive regulation of MIMO linear systems against unknown sinusoidal exogenous signals, Int. J. Adapt. Control Signal Process. 23 (2009) 581-603.

[14] X. Guo, M. Bodson, Analysis and implementation of an adaptive algorithm for the rejection of multiple sinusoidal disturbances, IEEE Trans. Control Syst. Technol. 17 (1) (2009).

[15] R. Marino, G.L. Santosuosso, Regulation of linear systems with unknown exosystems of uncertain order, IEEE Trans. Automat. Control 52 (2) (2007) 352-359.

[16] R. Marino, P. Tomei, Output regulation for linear systems via adaptive internal model, IEEE Trans. Automat. Control 48 (12) (2003) 2199-2202.

[17] V.O. Nikiforov, Adaptive servocompensation controller with an implicit reference model, Internat. J. Control 68 (2) (1997) 277-286.

[18] M. Bodson, S.C. Douglas, Adaptive algorithms for the rejection of periodic disturbances with unknown frequencies, Automatica 33 (12) (1997) 2213-2221.

[19] L. Hsu, R. Ortega, G. Damm, A globally convergent frequency estimator, IEEE Trans. Automat. Control 44 (4) (1999) 698-713.

[20] J. Huang, C.-F. Lin, On a robust nonlinear servomechanism problem, IEEE Trans. Automat. Control 39 (7) (1994) 1510-1513.

[21] A. Serrani, A. Isidori, L. Marconi, Semiglobal nonlinear output regulation with adaptive internal model, IEEE Trans. Automat. Control 46 (8) (2001) 1178-1194.

[22] Z. Ding, Global stabilization and disturbance suppression of a class of nonlinear systems with uncertain internal model, Automatica 39 (3) (2003) 471-479.

[23] Z. Chen, J. Huang, A general formulation and solvability of the global robust output regulation problem, IEEE Trans. Automat. Control 50 (4) (2005) 448-462.

[24] J.J. Fuchs, Estimating the number of sinusoids in additive white noise, IEEE Acoust. Speech Signal Process. 36 (12) (1988) 1846-1853. 